\title{
Anomalous Height Fluctuation Width in Crossover from Random to Coherent Surface Growths
}

\author{
K. Park and B. Kahng \\ Department of Physics and Center for Advanced Materials and Devices, \\ Kon-Kuk University, Seoul 143-701, Korea
}

\begin{abstract}
We study an anomalous behavior of the height fluctuation width in the crossover from random to coherent growths of surface for a stochastic model. In the model, random numbers are assigned on perimeter sites of surface, representing pinning strengths of disordered media. At each time, surface is advanced at the site having minimum pinning strength in a random subset of system rather than having global minimum. The subset is composed of a randomly selected site and its $(\ell-1)$ neighbors. The height fluctuation width $W^{2}(L ; \ell)$ exhibits the non-monotonic behavior with $\ell$ and it has a minimum at $\ell^{*}$. It is found numerically that $\ell^{*}$ scales as $\ell^{*} \sim L^{0.59}$, and the height fluctuation width at that minimum, $W^{2}\left(L ; \ell^{*}\right)$, scales as $\sim L^{0.85}$ in $1+1$ dimensions. It is found that the subset-size $\ell^{*}(L)$ is the characteristic size of the crossover from the random surface growth in the KPZ universality, to the coherent surface growth in the directed percolation universality.
\end{abstract}

PACS numbers: 68.35.Fx, 05.40.+j, 64.60.Ht

Recently subjects in the field of non-equilibrium surface growth have been attractive, which is due to the interest in theoretical classification of universality and also due to their applications to various physical phenomena such as crystal growth, molecular beam epitaxy, vapor deposition, and biological evolution, etc $[1]$. The interesting feature of non-equilibrium surface growth is the nontrivial scaling behavior of the height fluctuation width 2, i.e.,

$$
W^{2}(L, t)=\left\langle\frac{1}{L^{d^{\prime}}} \sum_{i}\left(h_{i}-\bar{h}\right)^{2}\right\rangle \sim L^{2 \alpha} f\left(t / L^{z}\right),
$$

where $h_{i}$ is the height of site $i$ on substrate. Here, $\bar{h}, L$, and $d^{\prime}$ denote mean height, system size, and substrate dimension, respectively. The symbol $\langle\cdots\rangle$ stands for statistical average. The scaling function behaves as $f(x) \rightarrow$ constant for $x \gg 1$, and $f(x) \sim x^{2 \beta}$ for $x \ll 1$ with $\beta=\alpha / z$. The exponents, $\alpha, \beta$ and $z$ are called the roughness exponent, the growth exponent, and the dynamic exponent, respectively.

The Kardar-Parisi-Zhang (KPZ) equation [3] was introduced to account for the effect of sideway growth, which is written as

$$
\frac{\partial h(x, t)}{\partial t}=\nu \nabla^{2} h(x, t)+\frac{\lambda}{2}(\nabla h(x, t))^{2}+\eta(x, t),
$$

where $\eta(x, t)$, called thermal noise, is assumed as white noise, $\langle\eta(x, t)\rangle=0$ and $\left\langle\eta(x, t) \eta\left(x^{\prime}, t^{\prime}\right)\right\rangle=2 D \delta^{d^{\prime}}(x-$ $\left.x^{\prime}\right) \delta\left(t-t^{\prime}\right)$ with noise strength $D$. Many stochastic models in the KPZ universality class have been introduced [1]. Among them, the restricted solid-on-solid (RSOS) model was introduced by Kim and Kosterlitz [4], satisfying the scaling relation, $\alpha_{t}+z_{t}=2$. The subscript means that the exponents are for thermal noise. In the RSOS model, a particle is deposited at a randomly selected site as long as the height difference $\Delta h$ between nearest neighbor columns remains as $\Delta h \leq 1$ even after deposition, otherwise, the particle is excluded from deposition. On the other hand, one may modify the dynamic rule of the RSOS model by replacing the exclusion by avalanche: At each time step, a particle is deposited at a randomly selected site, and avalanche may occur successively to nearest neighbor sites as long as the height difference between nearest neighbors, $\Delta h>1$. Then it was argued that this model also belongs to the KPZ universality class [5], but the model requires relatively large system size to see its asymptotic behavior. Let us call the former model the RSOS-A model, and the latter model the RSOS-B model. The surface-growth in the KPZ universality class is called the random surface-growth.

Physical properties of growing surface in disordered media are different from those of the thermal KPZ equation, Eq. (2). In order to account for the effect of disorder in porous media, quenched noise [6], which depends on position $x$ and height $h$, replaces thermal noise in Eq. (2). Then the quenched KPZ (QKPZ) equation is written as

$$
\frac{\partial h(x, t)}{\partial t}=\nu \nabla^{2} h(x, t)+\frac{\lambda}{2}(\nabla h(x, t))^{2}+\eta(x, h),
$$

where the noise satisfies, $\langle\eta(x, h)\rangle=0$, and $\left\langle\eta(x, h) \eta\left(x^{\prime}, h^{\prime}\right)\right\rangle=2 D \delta^{d^{\prime}}\left(x-x^{\prime}\right) \delta\left(h-h^{\prime}\right)$. Stochastic models associated with the QKPZ equation have been introduced [7,8]. The models show that the surface of the QKPZ equation in $1+1$ dimensions belongs to the directed percolation (DP) universality. The roughness exponent $\alpha_{q}$ in the QKPZ equation is given by the ratio of the correlation length exponents in perpendicular and parallel directions, $\nu_{\perp}$ and $\nu_{\|}$, of directed percolating clusters, which is $\alpha_{q}=\nu_{\perp} / \nu_{\|} \approx 0.63$. On the other hand, recently Sneppen introduced a stochastic model in which surface grows coherently [5]. In that model, random numbers, representing disorder of porous media, 
are assigned on each perimeter site of surface. Surface is advanced at the site having global minimum among the random numbers. The avalanche rule is then applied successively to nearest neighbor sites as long as $\Delta h>1$. Random numbers at the columns with increased heights are updated by new ones. The Sneppen model also belongs to the DP universality, and the roughness exponent is $\alpha_{s} \approx 0.63$ in $1+1$ dimensions [9]. The surface-growth of the Sneppen model is called the coherent surface-growth.

The coherent surface-growth model is closely related to the self-organized evolution model for biological systems [10]. In the evolution model, one considers random numbers assigned in one-dimensional array, which represent fitness of each species. Mutation of species is described in the model by updating random numbers. The updating occurs at the site having global minimum random number and its two nearest neighbors, and the random numbers at those sites are replaced by new ones at each time. Then as times go on, relatively small random numbers disappear by the updating process, and the distribution of random numbers exhibits a self-organized critical behavior. On the other hand, one may think of the situation where biological evolution is not motivated in globally optimized manner, but it may be driven by optimization within finite region out of entire system. This is analogous to the case that spin glass system is in meta-stable state within finite relaxation time rather than in globally stable state. Motivated by this idea, in this paper, we introduce a surface growth model in disordered media, where surface growth occurs at the site having the minimum random number in a subset of the entire system rather than having the global minimum random number. We think this model might be relevant to the case where the relaxation of surface growth in disordered media is not fast enough to spread into the whole system, so that surface growth is driven not in globally optimized manner, but in locally optimized manner.

To be specific, the model we consider in this paper is defined as follows: First, we consider one dimensional flat substrate with system size $L$. Random numbers are assigned on each site, which represent energy barriers (fitness) in the evolution model or pinning forces in the surface growth model by Sneppen. Second, we select a site randomly, and consider the subset composed of $\ell \equiv 2 r+1$ elements, the randomly selected site and its $2 r$ neighbor sites within distance $r$. It is worthwhile to note that the subset is regarded as a random sample because the site in the middle of the subset was selected at random. The subset is formed instantaneously, and its territory might overlap with subsequent one as shown in Fig. 1. Next, surface is advanced at the site having minimum random number among the $\ell$ elements in the subset, and the avalanche process is followed successively at its neighbor sites to keep the RSOS condition, $\Delta h \leq 1$, and it may spread out over the boundary of the subset. Finally, the random numbers at the sites with increased height are updated with new ones. The dynamic rule of the model is depicted in Fig. 1 . When $\ell=1$, this model corresponds to the RSOS-B model in the KPZ universality, whereas when $\ell=L$, it does to the Sneppen-B model in the DP universality. Accordingly one may see the crossover behavior from the KPZ limit to the DP limit as increasing $\ell$.

Since the roughness exponent $\alpha_{t}=1 / 2$ in the KPZ limit is smaller than the one $\alpha_{q} \approx 0.63$ in the DP limit, one may expect at a glance that the height fluctuation width $W^{2}(L ; \ell)$ in steady state increases monotonically with increasing $\ell$. However, we found the anomalous behavior numerically that $W^{2}(L ; \ell)$ decreases with increasing $\ell$ for small $\ell$, and increases for large $\ell$ as shown in Fig. 2. The minimum of $W^{2}(L ; \ell)$ becomes steeper and its location, $\ell^{*} / L$, which was rescaled by system size $L$, approaches to zero as $L$ increases. It is found that the location of the minimum scales as $\ell^{*} \sim L^{0.59}$, and the height fluctuation width at this minimum scales as $W^{2}\left(L ; \ell^{*}\right) \sim L^{0.85}$, which are shown in Figs. 3 and 4. The estimated values of $\ell^{*}$ and $W^{2}\left(L ; \ell^{*}\right)$ for different system sizes are tabulated below.

Table 1. Numerical values of the location $\ell^{*}$ and the height fluctuation width $W^{2}\left(L ; \ell^{*}\right)$ at the minimum for different system sizes.

\begin{tabular}{|c|c|c|}
\hline$L$ & $\ell^{*}$ & $W^{2}\left(L ; \ell^{*}\right)$ \\
\hline 64 & 11 & 5.9 \\
\hline 128 & 19 & 11.2 \\
\hline 256 & 27 & 20.3 \\
\hline 512 & 39 & 35.8 \\
\hline 1024 & 59 & 62.5 \\
\hline 2048 & 95 & 110.0 \\
\hline
\end{tabular}

The anomalous behavior may be attributed to the two effects, the random effect for small $\ell$ and the coherent effect for large $\ell$. For small $\ell$, surface grows by random deposition with avalanches, and it belongs to the KPZ universality. Thus the height fluctuation width depends on system size as $W^{2}(L ; \ell) \sim L^{2 \alpha_{t}}$ with $2 \alpha_{t}=1$, however, it would also depend on subset size $\ell$. In order to find out the $\ell$-dependent behavior of $W^{2}(L ; \ell)$ in phenomenological level, we plot $W^{2}(L ; \ell)$ versus $\ell$ in double logarithmic scales for several values of $L$ as shown in Fig. 5. The slopes are measured to be $\approx-0.2$ for large system sizes $L=1024$ and 2048. Based on this measurement, $W^{2}(L ; \ell)$ is written as $W^{2}(L ; \ell) \sim \ell^{-0.2} L$ for small $\ell$. This result is contrary to the one, based on the coarsegraining scaling argument, $W^{2}(L ; \ell) \sim(L / \ell)^{2 \alpha_{t}} \ell^{2 \alpha_{q}}$, which exhibits the increasing behavior of $W^{2}$ with increasing $\ell$. On the other hand, when $\ell$ is large enough, surface updating is initiated mainly at the site having global minimum random number of entire system. When the site of global minimum is selected, which occurs with probability $\ell / L$, the surface becomes correlated by the Sneppen dynamics, however, when the site of the global 
minimum is not selected, the correlation formed by the Sneppen's dynamics is relaxed. Thus, when $\ell$ is large enough so that the contribution by the Sneppen dynamics is sufficiently dominant, one may write the dominant term of the averaged height fluctuation width as $W^{2}(L ; \ell) \sim\left(\frac{\ell}{L}\right) L^{2 \alpha_{q}}$. That is because the statistical average was taken over the quantity of the square of meanheight deviation in Eq. (1). Combining the two asymptotic behaviors obtained in phenomenological level, the height fluctuation width is written as

$$
W^{2}(L ; \ell) \sim \ell^{-0.2} L+\left(\frac{\ell}{L}\right) L^{1.26} .
$$

The two terms exhibit the competing behavior with respect to $\ell$, which yields the anomalous behavior. Taking the derivative with respect to $\ell$, the location $\ell^{*}$ of the minimum is obtained as that $\ell^{*} \sim L^{0.62}$, which is close to the numerical measurement, $\ell^{*} \sim L^{0.59}$. We also examine the size-dependent behavior of the height fluctuation width at the minimum by plugging $\ell^{*}$ into Eq. (4), and obtain that $W^{2}\left(L ; \ell^{*}\right) \sim L^{0.88}$. This result is also close to the numerical measurement, $W^{2}\left(L ; \ell^{*}\right) \sim L^{0.85}$. The numerical estimations for $\ell^{*}$ and $W^{2}\left(L ; \ell^{*}\right)$ are better explained by minimizing the formula,

$$
W^{2}(L ; \ell) \sim\left(\frac{\ell^{2 \alpha_{t}}}{\ell^{2 \alpha_{q}}}\right) L^{2 \alpha_{t}}+\left(\frac{\ell}{L}\right) L^{2 \alpha_{q}},
$$

however, the derivation of this formula is not clear.

In order to understand physical meaning of the characteristic subset-size $\ell^{*}$, we plot $W^{2}(L ; \ell)$ versus $L$ up to $L=2048$ in double logarithmic scales for typical subset sizes, $\ell=5$ and 41 in Fig. 6 . The size of $\ell=5$ corresponds to the case where it is smaller than $\ell^{*}(L)$ for all system sizes $L$ used in Fig. 6 . However, the size of $\ell=41$ does to the case where it is smaller than $\ell^{*}(L)$ in part for $L=1024$ and 2048, close to $\ell^{*}(L)$ for $L=512$, but larger than $\ell^{*}(L)$ in part for $L=64$ and 128. For the case of $\ell=5$, all data are on a straight line, whereas, for the case of $\ell=41$, forming a straight line breaks down for relatively smaller system sizes $L=64$ and 128. Fig. 6 suggests that $\ell^{*}(L)$ be the characteristic subset-size such that when $\ell<\ell^{*}(L)$, the roughness of overall surface is determined by random effect, whereas when $\ell>\ell^{*}(L)$, it is done by coherent effect. Accordingly, the subset size $\ell^{*}$ has the meanings of not only the location of the minimum of the anomalous height fluctuation width, but also the critical size at which the crossover from random to coherent surface-growths occurs. Also note that even for the case of $\ell>\ell^{*}(L)$, the surface height fluctuation width does not behave as $W^{2}(L ; \ell) \sim L^{2 \alpha_{q}}$. The roughness exponent has smaller value than $2 \alpha_{q}$ as appeared in Fig. 6, and the numerical estimation of the roughness exponent is likely to be $2 \alpha_{q}-1$ as Eq. (4) for fixed $\ell$. The behavior of $W^{2}(L ; \ell) \sim L^{2 \alpha_{q}}$ occurs when $\ell$ also increases as $L$ increases. In thermodynamic limit, $L \rightarrow \infty$, the characteristic size $\ell^{*}$ goes to infinity, so that for finite $\ell$, the roughness of overall surface is determined by random effect, and the surface is described in the KPZ universality. Next, we examine the height-height correlation function,

$$
C(r ; L, \ell) \equiv\left\langle\frac{1}{L} \sum_{x}(h(x+r)-h(x))^{2}\right\rangle,
$$

which is defined for fixed $L$, and $\ell$. In Fig. 7 , we plot $C(r) / l^{2 \alpha_{q}}$ versus $(r / \ell)$ in double logarithmic scales for several values of $\ell$, and $L=2048$. In Fig. 7, the data are well collapsed for $r / \ell<1$, while they are not collapsed for $r / \ell>1$. Accordingly, the coherent surface growth occurs within the range of $r<\ell$, however, the roughness of overall surface is determined by the criterion depending on $\ell^{*}(L)$ and $L$ above.

It would be interesting to study the $\ell$-dependent behavior of dynamic properties of the height fluctuation width $W^{2}(L, t ; \ell)$. The study is based on numerical simulations for fixed system size, say, $L=1024$. As shown in inset of Fig. 8, there exist four distinct regimes for $W^{2}(L, t ; \ell)$. In the first regime, $W^{2}(L, t ; \ell)$ increases according to the Poisson distribution, and $W^{2}(L, t ; \ell) \sim t^{2 \beta_{1}}$ with $2 \beta_{1}=1$. The first regime terminates at $t_{1}$, which is independent of subset-size $\ell$. In the second regime, surface becomes correlated by the coherent effect, which is caused by the selection of minimum random number in selected subset, however, the decorrelation also occurs simultaneously by the random effect, which is caused by the selection of the random subset. Since the value of the dynamic exponent $z_{s}=0.63$ for the Sneppen dynamics is smaller than the one $z_{t}=1.5$ for the KPZ dynamics, the coherent effect spreads faster than the random effect in early times. Thus the growth exponent $2 \beta_{2}$ in the second regime has the value more likely close to the Sneppen value, $2 \beta_{s}=2$, however, the value is a little bit smaller by the decorrelation by the random effect. The growth exponent $2 \beta_{2}$ depends on subset-size $\ell$ as tabulated in Table 2. Based on the measurement in Fig. 9, the growth exponent is likely to depend on $\ell$ as $2 \beta_{2} \sim(0.1) \log \ell$ for $\ell<\ell^{*}$, however for $\ell>\ell^{*}$, the value of the growth exponent is expected to be close to the one of the Sneppen dynamics as shown in Fig. 8. The second regime terminates at $t_{2}$. In Fig. 10, the threshold times are likely to scale as $t_{2} \sim \ell^{0.37}$ for $\ell<\ell^{*}$. The values of the height fluctuation width at the threshold value $t_{2}$ are likely to scale as $W_{2}^{2}\left(L, t_{2} ; \ell\right) \sim \ell^{0.92}$ as shown in Fig. 11. In the third regime, the random effect appears much dominantly, and the coherence of surface formed in the second regime becomes decorrelated in this regime. As subset size is smaller, the third regime is much dominant, and the growth exponent $\beta_{3}$ becomes much closer to the KPZ value, whereas as subset size is larger, the decorrelation effect becomes much weaker, so that the growth exponent $\beta_{3}$ becomes smaller. It is likely that $2 \beta_{3} \sim(-0.37) \log \ell$ as shown in Fig. 12. The third regime terminates at $t_{3}$. The numerical values of $t_{3}$ for different sizes of $\ell$ locate too closely for small $\ell$ to be measured 
numerically.

Table 2. Numerical estimation for the values of the growth exponents, the threshold times, and the height fluctuation widths for various subset sizes $\ell$.

\begin{tabular}{|c|c|c|c|c|}
\hline$\ell$ & $2 \beta_{2}$ & $\log _{10}\left(t_{2}\right)$ & $\log _{10}\left(W_{2}^{2}\right)$ & $2 \beta_{3}$ \\
\hline 3 & 1.12 & -0.10 & 0.20 & 0.66 \\
\hline 5 & 1.16 & -0.10 & 0.20 & 0.64 \\
\hline 9 & 1.23 & 0.00 & 0.33 & 0.60 \\
\hline 17 & 1.31 & 0.10 & 0.55 & 0.50 \\
\hline 33 & 1.40 & 0.20 & 0.80 & 0.38 \\
\hline 65 & 1.59 & 0.31 & 1.12 & 0.28 \\
\hline 129 & 1.68 & 0.42 & 1.60 & 0.17 \\
\hline 257 & 1.78 & 0.60 & 1.80 & 0.17 \\
\hline
\end{tabular}

We also investigated the $\ell$-dependent behavior of the distribution of random numbers after reaching saturated state. As shown in Fig. 13, the distribution is flat for $\ell=1$, and exhibits a critical behavior for $\ell=L$. Between the two limits, the distributions look like a rounded step function. It would be interesting to note that all distribution functions for different $\ell$ pass through a specific value of random number $B_{c}$, which corresponds to the threshold of the self-organized critical state [9]. The value of $B_{c}$ equals to $1-P_{c}=0.462$, where $P_{c}$ is the directed percolation threshold.

Recently, the crossover behavior from the random surface growth to the coherent surface growth was considered by Vergeles [11. In that study, surface growth occurs at site $x$ on substrate with the probability, $P(x) \sim e^{-q(x) / T}$, where $q(x)$ is a random pinning strength of site $x$, and is also updated with height advance. $T$ is temperature. It was found that the surface of the model reduces to the one of the Sneppen dynamics when $T=0$, however, for $T \neq 0$, it does to the one of the KPZ universality. The temperature $T$ plays a role of tuning parameter for the crossover behavior. However, since the tuning parameter is in the form of exponential function, it is hard to see finite size dependent behavior of the crossover which is very sensitive to tuning the parameter as we studied in this paper. Nevertheless, the anomalous behavior may be observed barely in the plot of $W(L ; T)$ versus $L$ for different temperatures, Fig. 1 (b) in Ref. 11, where the curves of $W(L, T)$ cross to each other, However the crossover behavior has not been remarked in Ref. 11.

In summary, we have introduced a stochastic model for surface growth, which is a generalization of the restricted solid-on-solid model in the KPZ universality and the Sneppen model in the directed percolation limit, and have investigated the crossover of the two limits. Deposition occurs at the site having minimum of random numbers within finite subset rather than of entire system. The subset is composed of $\ell$ elements, a randomly selected site and its $\ell-1$ neighbors. Changing subset-size $\ell$, the height fluctuation width exhibits the anomalous behavior having a minimum. The anomalous behavior is due to the two competing effects, the random effect for small $\ell$ and the coherent effect for large $\ell$. The minimum of the surface height fluctuation width, locating at $\ell^{*} \sim L^{0.59}$, is scaled as $W^{2}\left(L ; \ell^{*}\right) \sim L^{0.85}$ in $1+1$ dimensions. The characteristic subset size $\ell^{*}(L)$ has the meaning that for $\ell<\ell^{*}(L)$, the surface grows randomly and belongs to the KPZ universality, whereas for the opposite case, surface grows coherently. The dynamic properties of the crossover have also been investigated. In early stage of growth, surface becomes correlated according to the Sneppen dynamics, and in late stage, the surface correlation is relaxed by the random process. The phenomenon of the dynamic correlation-decorrelation behavior also appears in a stochastic model [12] for the flux line dynamics with transversal and longitudinal fluctuations, which might be described by the coupled quenched KPZ equation 13].

One of the authors (B.K.) thanks Drs. A.-L. Barabási and D. Dhar for helpful discussions. This work is supported in part by the NON DIRECTED RESEARCH FUND, the Korea Research Foundation, in part by the BSRI program of Ministry of Education, Korea, and in part by the KOSEF through the SRC program of SNUCTP.

[1] For a recent review, see, for examples, F. Family and T. Vicsek, Dynamics of Fractal Surfaces (World Scientific, Singapore, 1991); A.-L. Barabási and H. E. Stanely, Fractal Concepts in Surface Growth (Cambridge University Press, Cambridge, England, 1995).

[2] F. Family, J. Phys. A 19, L441 (1986).

[3] M. Kardar, G. Parisi, and Y. Zhang, Phys. Rev. Lett. 56, 889 (1986).

[4] J. M. Kim and J. M. Kosterlitz, Phys. Rev. Lett. 62, 2289 (1989).

[5] K. Sneppen, Phys. Rev. Lett. 69, 3539 (1992).

[6] D. Kessler, H. Levine, and Y. Tu, Phys. Rev. A 43, 4551 (1991).

[7] S. V. Buldyrev, A.-L. Barabási, F. Caserta, S. Havlin, H. E. Stanley and T. Viseck, Phys. Rev. A 45, R8313 (1992).

[8] L.-H. Tang and H. Leschhorn, Phys. Rev. A 45 R8309 (1992).

[9] Z. Olami, I. Procaccia, and R. Zeitak, Phys. Rev. E 49, 1232 (1994); H. Leschhorn and L.-H. Tang, Phys. Rev. E 49, 1238 (1994).

[10] P. Bak and K. Sneppen, Phys. Rev. Lett. 71, 4083 (1993); H. Flyvberg, K. Sneppen, and P. Bak, ibid 71, 4087 (1993). 
[11] M. Vergeles, Phys. Rev. Lett. 75, 1969 (1995).

[12] B. Kahng and K. Park (unpublished).

[13] D. Ertas and M. Kardar, Phys. Rev. B 53, 3520 (1996).
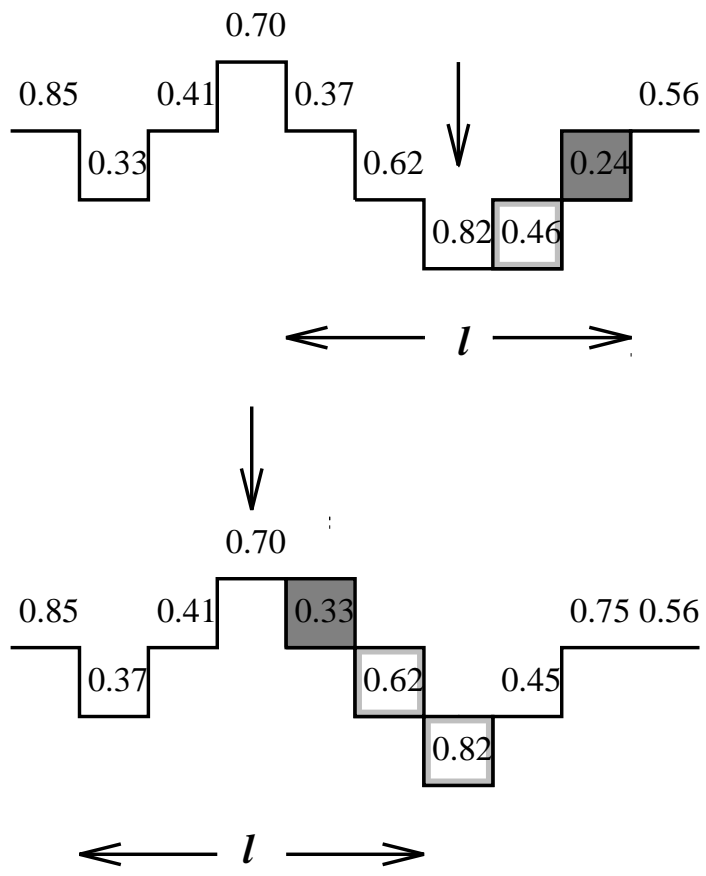

FIG. 1. Schematic representation of the stochastic rule. The sites arrowed are randomly selected sites. The dark squares denote the sites of minimum random number within subset sized $\ell=5$. The white squares denote the sites updated by avalanches. The subset could overlap with the one (lower one) of subsequent time.

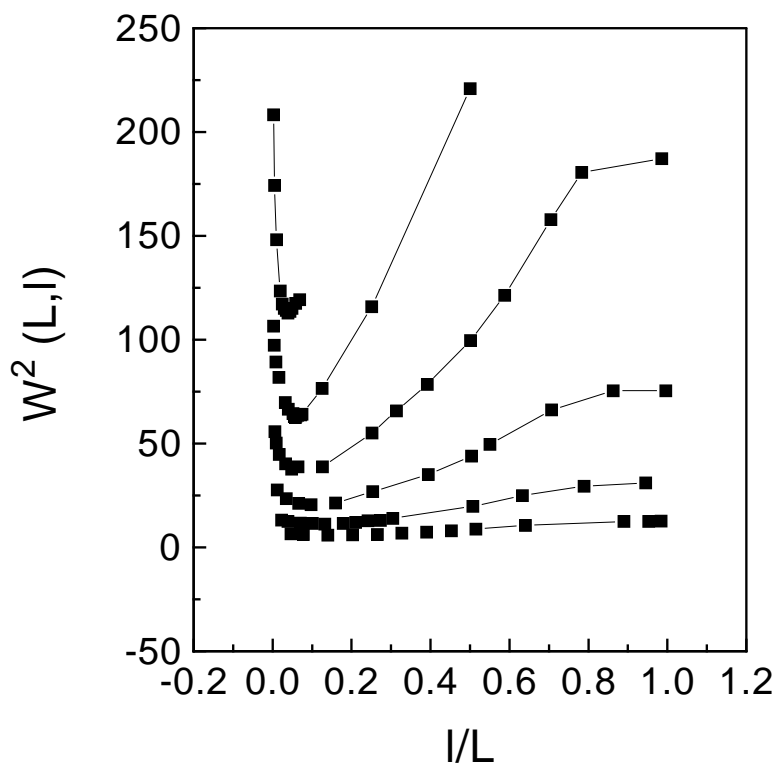

FIG. 2. Plot of the surface height fluctuation widths $W^{2}(L ; \ell)$ versus subset size $\ell / L$ rescaled by system size $L$ in steady state. Numerical data are for system sizes $L=64,128,256,512,1024$ and 2048 from the bottom.

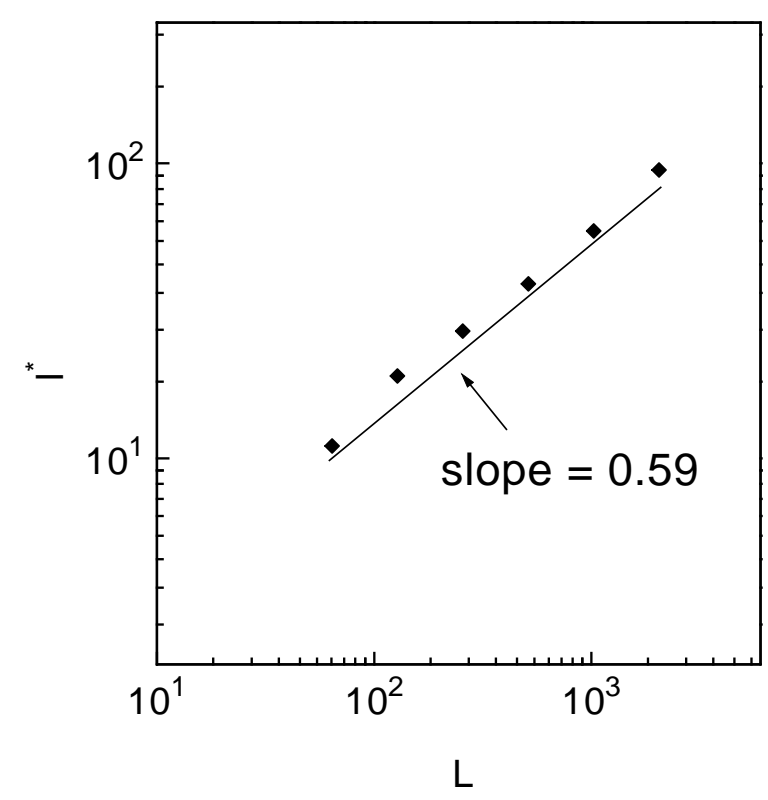

FIG. 3. Double-logarithmic plot of the estimated location $\ell^{*}$ of the minimum versus system size $L$. The data are for $L=64,128,256,512,1024$, and 2048 .

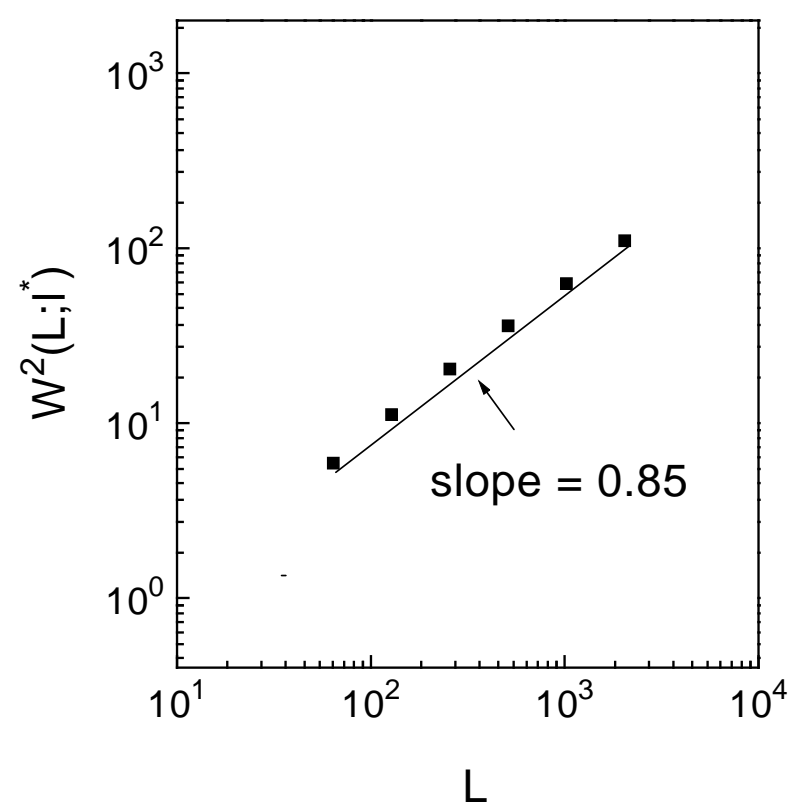


FIG. 4. Double-logarithmic plot of the surface height fluctuation width at the minimum position $W^{2}\left(L ; \ell^{*}\right)$ versus system size $L$. The data are for $L=64,128,256,512,1024$, and 2048 .

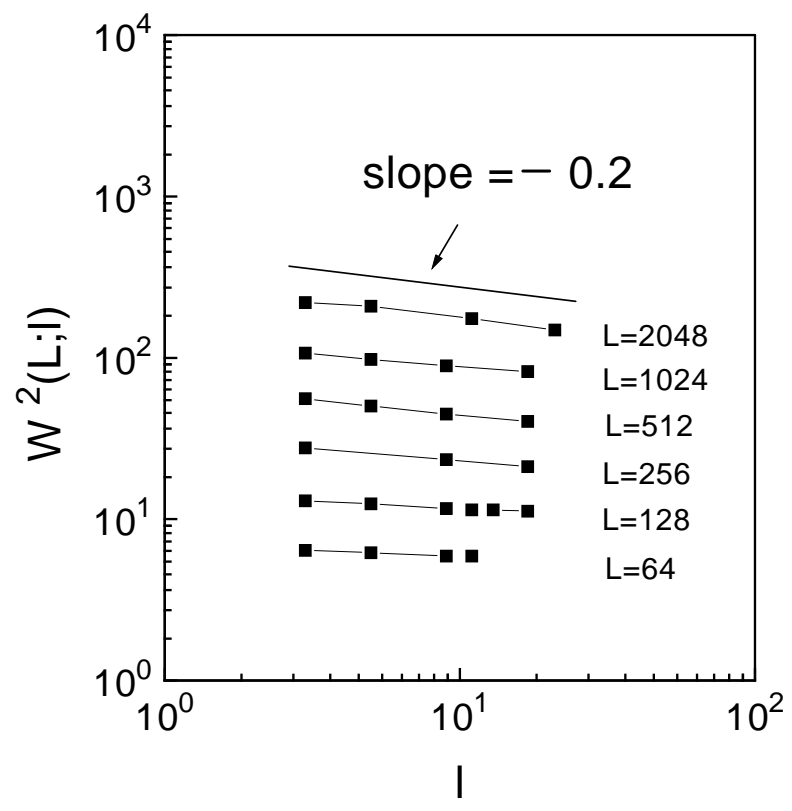

FIG. 5. Double-logarithmic plot of $W^{2}(L ; \ell)$ versus $\ell$ for various system sizes. The data seem to be on straight lines with slope -0.2 .

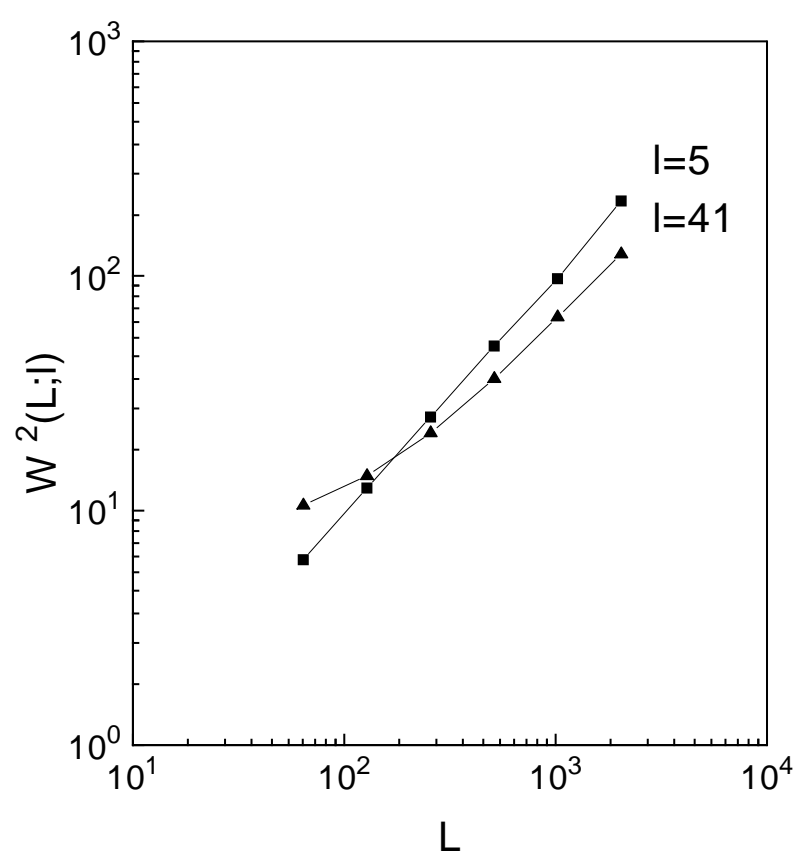

FIG. 6. Double-logarithmic plot of $W^{2}(L ; \ell)$ versus $L$ for typical subset sizes $\ell=5$ and 41 .

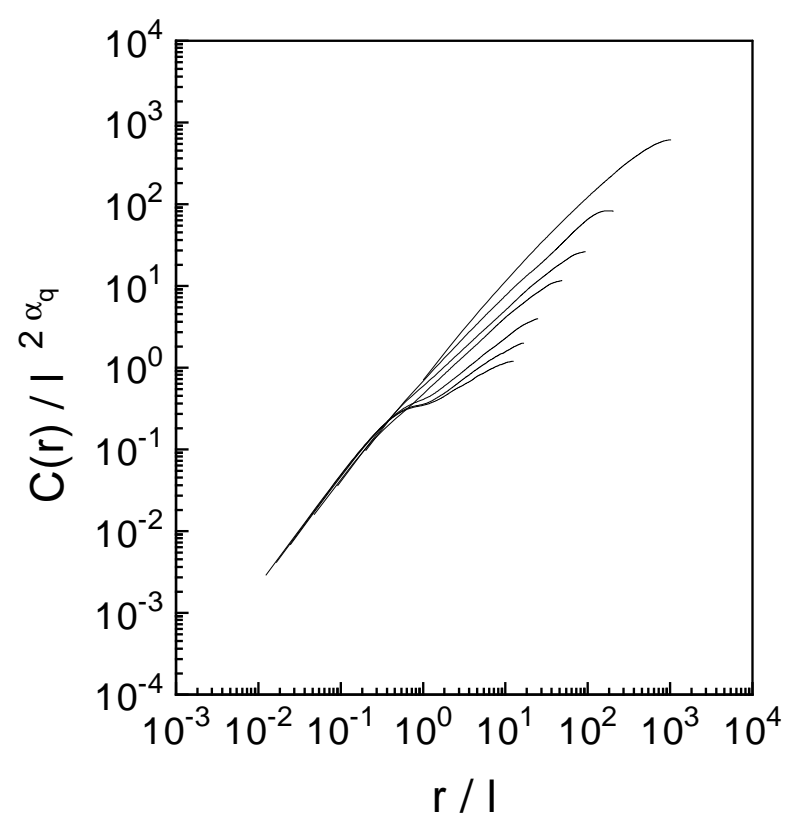

FIG. 7. Double-logarithmic plot of $C(r) / \ell^{2 \alpha_{q}}$ versus $r / \ell$ for subset sizes $\ell=5,11,21,41,61,81$ and 141 . The data are for system size $L=2048$.

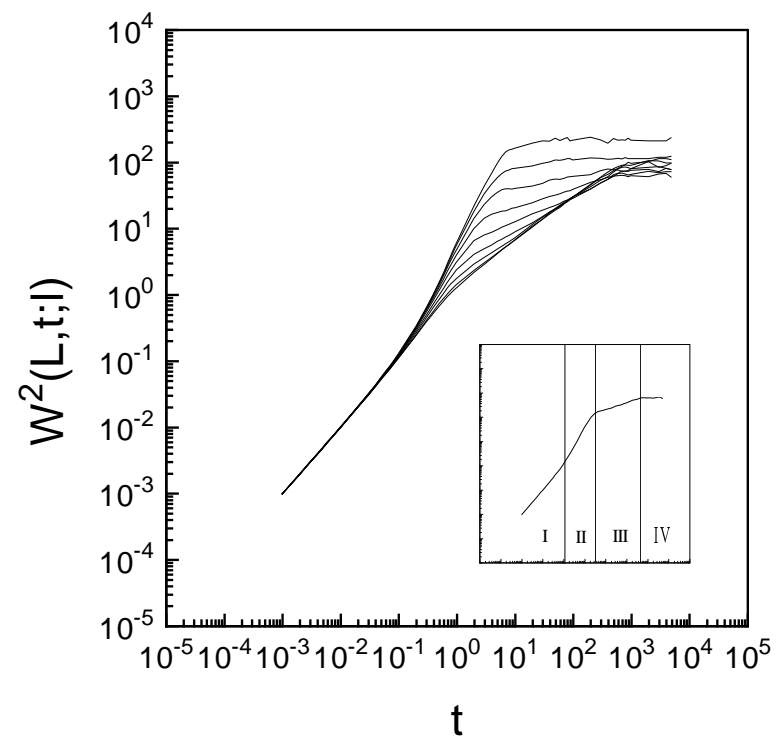

FIG. 8. Double-logarithmic plot of $W^{2}(L, t ; \ell)$ versus time $t$ for system size $L=1024$. The data are for subset sizes $\ell=3,5,9,17,33,65,129,257$ and 513. Inset: Distinct four regimes are observed for the case of $L=1024$ and $l=65$. 


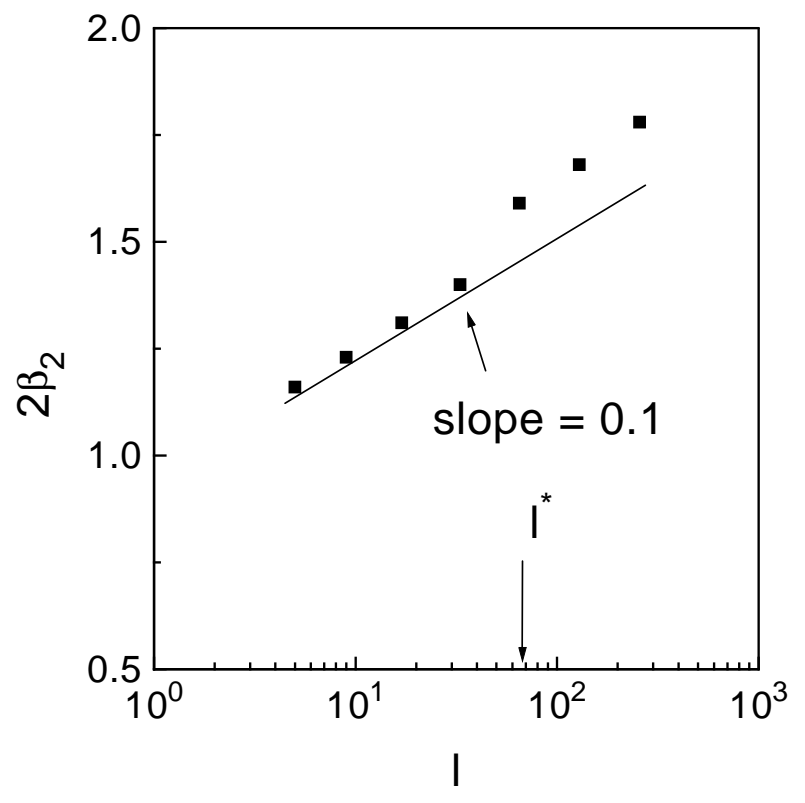

FIG. 9. Semi-logarithmic plot of $2 \beta_{2}$ versus $\ell$ for $L=1024$. The data seem to be on a straight line with slope 0.1 up to the characteristic subset size $\ell^{*}$.

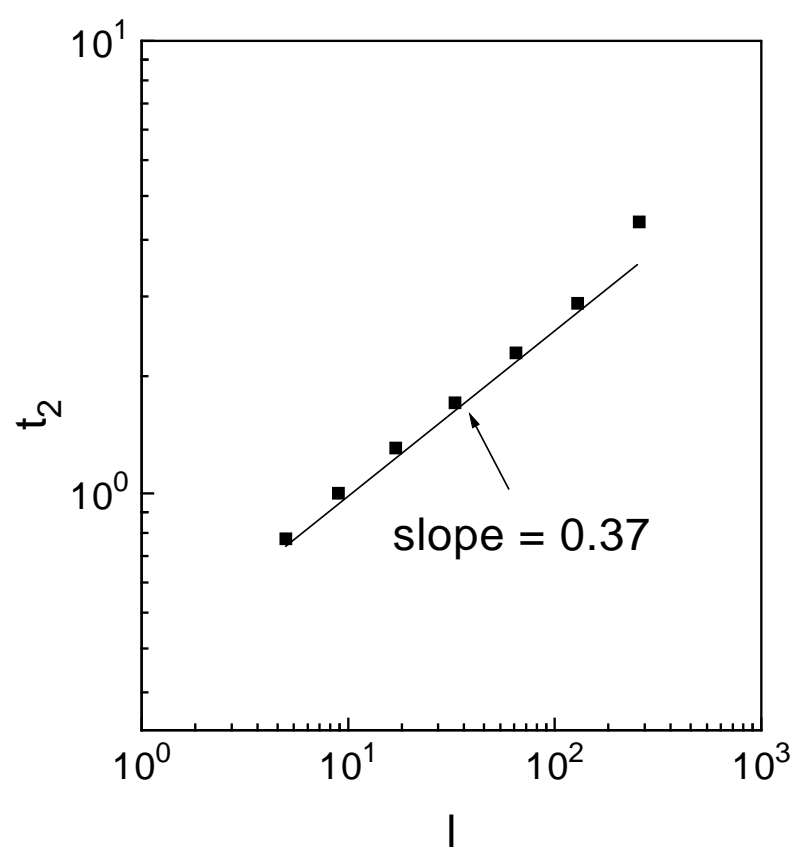

FIG. 10. Double-logarithmic plot of $t_{2}$ versus $\ell$ for $L=1024$. The data seem to be on a straight line with slope 0.37 .

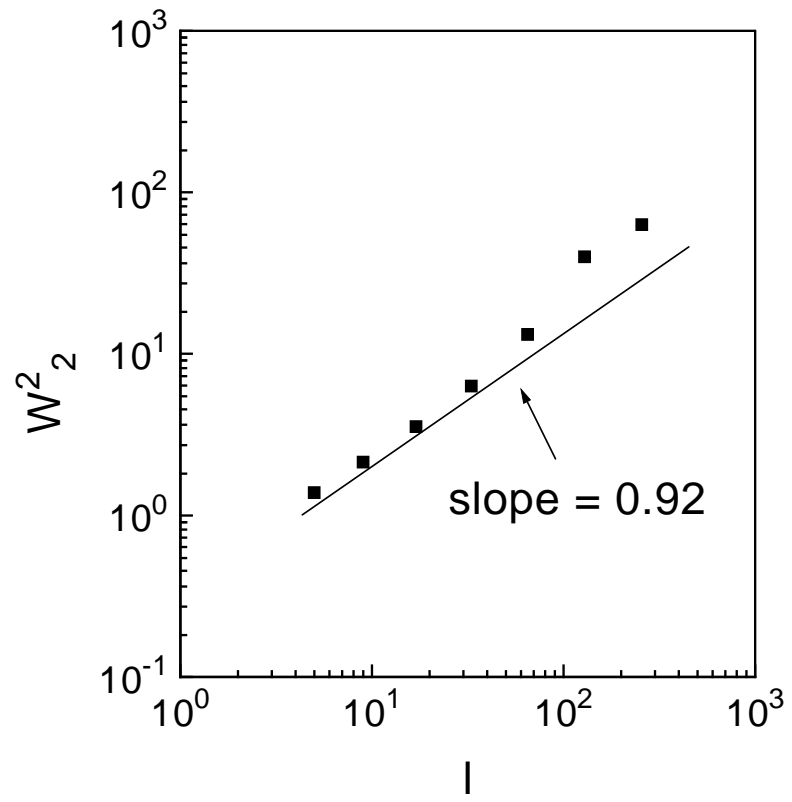

FIG. 11. Double-logarithmic plot of ${W_{2}}^{2}$ versus $\ell$ for $L=1024$. The data seem to be on a straight line with slope 0.92 up to the characteristic subset size $\ell^{*}$.

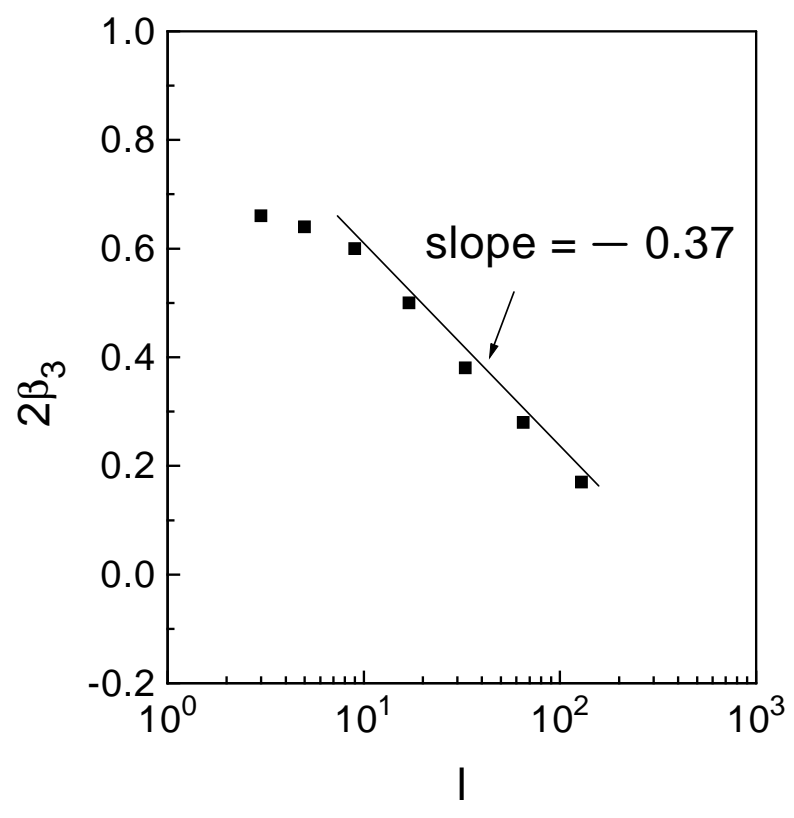

FIG. 12. Semi-logarithmic plot of $2 \beta_{3}$ versus $\ell$ for $L=1024$. The line has the slope -0.37 , and the data are for $\ell=3,5,9,17,33,65$ and 129 , respectively. 


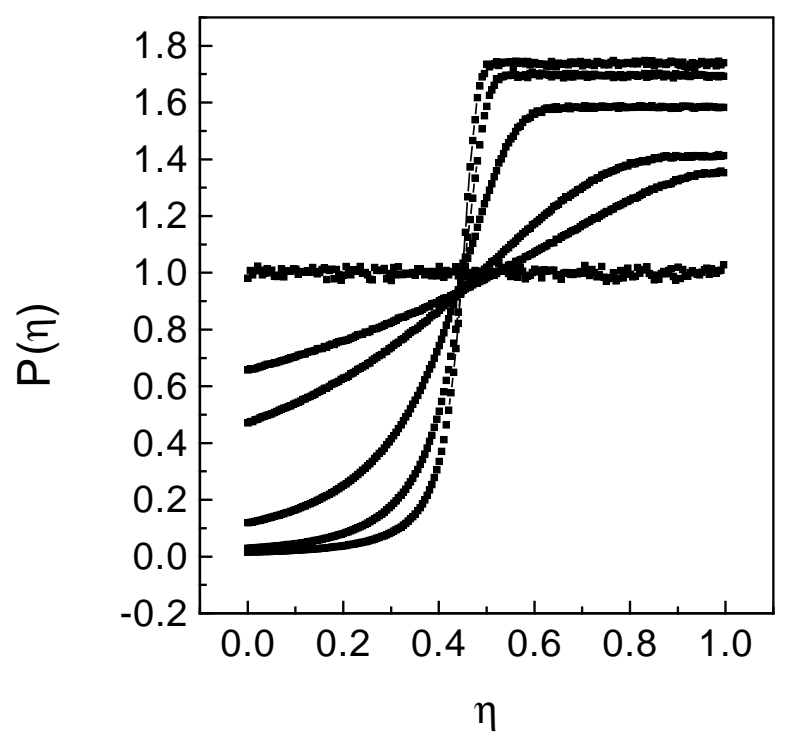

FIG. 13. Plot of the random number distribution after saturation for $L=256$. The data are for $\ell=1,3,5,27,129$ and 256 , respectively. 